TAIWANESE JOURNAL OF MATHEMATICS

Vol. 14, No. 4, pp. 1283-1296, August 2010

This paper is available online at http://www.tjm.nsysu.edu.tw/

\title{
WEIGHTED APPROXIMATION BY $q$-SZÁSZ-KING TYPE OPERATORS
}

\author{
Octavian Agratini and Ogun Doğru
}

\begin{abstract}
By using $q$-calculus, in the present paper we construct Szász type operators in King sense, this meaning the operators preserve the first and the third test function of Bohman-Korovkin theorem. Rate of local and global convergence is obtained in the frame of weighted spaces. The statistical approximation property of our operators is also revealed.
\end{abstract}

\section{INTRODUCTION}

During the last decade, $q$-calculus was intensively used in the construction for different generalizations of many classical sequences of linear positive operators. The pioneer work has been made by A. Lupaş [18] and G. M. Phillips [21] who proposed generalizations of Bernstein polynomials based on the $q$-integers. Numerous properties of these remarkable polynomials have been extended to their $q$-analogues. We refer, for instance, to the results of S. Ostrovska [19], [20], M. M. Derriennic [7], V.S. Videnskii [23], H. Wang [24]. Other important classes of discrete operators have been investigated by using $q$-calculus. For example: $q$ Meyer-König and Zeller operators appear in the papers of T. Trif [22], O. Doğru and O. Duman [8]; $q$-Bleimann, Butzer and Hahn operators have been studied by A. Aral and O. Doğru [4]; $q$-Szász Mirakjan operators are investigated by A. Aral and V. Gupta [5].

It is also known that J.P. King [15] has presented an unexpected example of operators of Bernstein type which preserve the test functions $e_{0}$ and $e_{2}$ of BohmanKorovkin theorem. We recall that the three test functions of this criterion, usually denoted by $e_{k}$, are the monomials $e_{k}(x)=x^{k}, k=0,1,2$. A general technique to construct sequences of operators of discrete type with the same property as in

Received May 8, 2008, accepted August 18, 2008.

Communicated by Sen-Yen Shaw.

2000 Mathematics Subject Classification: 41A36, 41A25.

Key words and phrases: $q$-integers, Positive linear operators, Statistical convergence, Weighted modulus of smoothness. 
King's example was presented by the first author [2]. In the same paper particular classes such as Szász-Mirakjan, Baskakov, Bernstein-Chlodovsky operators, have been modified in King's sense.

The goal of this article is to construct and investigate a variant of Szász operators based on $q$-calculus and King's model. The paper is organized as follows. Section 2 contains some basic facts regarding both $q$-calculus and statistical convergence. The construction of the announced class of operators is also presented. Section 3 deals with statistical approximation property of our operators in a weighted certain space. Section 4 centers around the rate of local and global convergence of our sequence for functions with polynomial growth. The main tool is a weighted modulus of smoothness.

\section{Notation AND PReLIMINARIES}

For the reader's convenience and to make the exposition self-contained, we collect information regarding two concepts: $q$-calculus and statistical approximation.

Set $\mathbb{N}_{0}=\{0\} \cup \mathbb{N}$ and $\mathbb{R}_{+}=[0, \infty)$. Throughout the paper we shall assume that $q \in(0,1)$. Following the definitions and notations of [14, pp. 7-13], for any real number $a$ and $x$ we set

$$
[a]_{q}=\frac{1-q^{a}}{1-q}, \quad(1-x)_{q}^{a}=\prod_{j=0}^{\infty} \frac{1-q^{j} x}{1-q^{j+a} x} .
$$

As special case we consider $[a]_{1}=a$. For the integer $n \geq 1$, the $q$-integer is $[n]_{q}=1+q+\cdots+q^{n-1}$. One also takes place $(1-x)_{q}^{n}=\prod_{j=0}^{n-1}\left(1-q^{j} x\right)$. The $q$-factorial $[n]_{q}$ ! of the element $n \in \mathbb{N}$ means $[n]_{q} !=[1]_{q}[2]_{q} \ldots[n]_{q}$. Set $[0]_{q} !=1$. Also, the $q$-binomial or the Gaussian coefficients are denoted by $\left[\begin{array}{l}n \\ k\end{array}\right]_{q}$ and are defined by

$$
\left[\begin{array}{l}
n \\
k
\end{array}\right]_{q}=\frac{[n]_{q} !}{[k]_{q} ![n-k]_{q} !}, \quad k=0,1, \ldots, n
$$

Clearly, $[n]_{1} !=n$ ! and $\left[\begin{array}{l}n \\ k\end{array}\right]_{1}$ represents $\left(\begin{array}{l}n \\ k\end{array}\right)$, the ordinary binomial coefficients. Two different $q$-expansions named $E_{q}$ and $\varepsilon_{q}$ of the exponential function $x \mapsto e^{x}$ are given as follows (see [13, p. 9] or [14, pp. 30-31])

$$
\begin{aligned}
& E_{q}(x)=\sum_{k=0}^{\infty} q^{k(k-1) / 2} \frac{x^{k}}{[k]_{q} !}, \quad x \in \mathbb{R}, \\
& \varepsilon_{q}(x)=\sum_{k=0}^{\infty} \frac{x^{k}}{[k]_{q} !}, \quad|x|<\frac{1}{1-q} .
\end{aligned}
$$


With the help of the notation $(1-a)_{q}^{\infty}=\prod_{j=0}^{\infty}\left(1-q^{j} a\right)$, it is proved that

$$
E_{q}(x)=(1+(1-q) x)_{q}^{\infty}, \quad \varepsilon_{q}(x)=\frac{1}{(1-(1-q) x)_{q}^{\infty}}
$$

and, consequently, the following relation between $q$-exponential functions

$$
E_{q}(x) \varepsilon_{q}(-x)=1, \quad|x|<\frac{1}{1-q},
$$

holds. We mention that these $q$-analogues of the classical exponential functions are valid for each $q \in(-1,1)$. Moreover, $\lim _{q \rightarrow 1^{-}} E_{q}(x)=\lim _{q \rightarrow 1^{-}} \varepsilon_{q}(x)=e^{x}$.

Further on, let recall the concept of statistical convergence. The density of a set $K \subset \mathbb{N}$ is defined by

$$
\delta(K)=\lim _{n \rightarrow \infty} \frac{1}{n} \sum_{k=1}^{n} \chi_{K}(k),
$$

provided the limit exists, where $\chi_{K}$ is the characteristic function of $K$. Clearly, the sum of the right hand side represents the cardinality of the set $\{k \leq n: k \in K\}$. Following [10], a sequence $x=\left(x_{k}\right)_{k \geq 1}$ is statistically convergent to a real number $L$ if, for every $\varepsilon>0$,

$$
\delta\left(\left\{k \in \mathbb{N}:\left|x_{k}-L\right| \geq \varepsilon\right\}\right)=0 .
$$

In this case we write $s t-\lim _{n} x_{n}=L$. It is known that any convergent sequence is statistically convergent, but not conversely. Closely related to this notion is Astatistical convergence, where $A=\left(a_{n, k}\right)$ is an infinite summability matrix. For a given sequence $x=\left(x_{k}\right)_{k \geq 1}$, the A-transform of $x$ denoted by $(A x)_{n}, n \in \mathbb{N}$, is defined by

$$
(A x)_{n}=\sum_{k=1}^{\infty} a_{n, k} x_{k}, \quad n \in \mathbb{N},
$$

provided the series converges for each $n$. Suppose that $A$ is non-negative regular summability matrix. Then $x$ is A-statistically convergent to the real number $L$ if, for every $\varepsilon>0$, one has

$$
\lim _{n \rightarrow \infty} \sum_{k \in I(\varepsilon)} a_{n, k}=0
$$

where $I(\varepsilon)=\left\{k \in \mathbb{N}:\left|x_{k}-L\right| \geq \varepsilon\right\}$. We write $s t_{A}-\lim _{n} x=L$, see e.g. [11], [16].

In approximation theory by linear positive operators, the statistical convergence has been examined for the first time by A.D. Gadjiev and C. Orhan [12]. 
In order to introduce a $q$-variant for Szász-Mirakjan operators, right to the start we present a construction due to A. Aral [3] and studied in deepness by A. Aral and V. Gupta [5]. Let $\left(b_{n}\right)_{n \geq 1}$ be a sequence of positive numbers such that $\lim _{n} b_{n}=\infty$. For each $n \in \mathbb{N}, q \in(0,1)$ and $f \in C\left(\mathbb{R}_{+}\right)$the authors defined

$$
\left(S_{n}^{q} f\right)(x)=E_{q}\left(-[n]_{q} \frac{x}{b_{n}}\right) \sum_{k=0}^{\infty} f\left(\frac{[k]_{q} b_{n}}{[n]_{q}}\right) \frac{\left([n]_{q} x\right)^{k}}{[k]_{q} ! b_{n}^{k}},
$$

where $0 \leq x<\frac{b_{n}}{1-q^{n}}$. The following explicit expressions for $S_{n}^{q} e_{k}, k=0,1,2$, have been established [3, Eqs. (3.5)-(3.7)]

$$
S_{n}^{q} e_{0}=e_{0}, \quad S_{n}^{q} e_{1}=e_{1}, \quad S_{n}^{q} e_{2}=q e_{2}+\frac{b_{n}}{[n]_{q}} e_{1} .
$$

In [2] the classical Szász-Mirakjan operators have been modified in King's sense. Following a similar route, we transform the operators defined at (2.3) in order to preserve the quadratic function $e_{2}$. Defining the functions

$$
v_{n, q}(x)=\frac{1}{2 q[n]_{q}}\left(-b_{n}+\sqrt{b_{n}^{2}+4 q[n]_{q}^{2} x^{2}}\right), \quad x \geq 0,
$$

we consider the linear and positive operators

$$
\left(S_{n, q}^{*} f\right)(x)=E_{q}\left(-[n]_{q} \frac{v_{n, q}(x)}{b_{n}}\right) \sum_{k=0}^{\infty} f\left(\frac{[k]_{q} b_{n}}{[n]_{q}}\right) \frac{\left([n]_{q} v_{n, q}(x)\right)^{k}}{[k]_{q} ! b_{n}^{k}}
$$

where $x \in J_{n}(q):=\left[0, \frac{b_{n}}{1-q^{n}}\right)$.

Lemma 2.1. The operators defined at (2.5) verify for each $x \in J_{n}(q)$ the following identities

$$
\begin{gathered}
\left(S_{n, q}^{*} e_{0}\right)(x)=1, \quad\left(S_{n, q}^{*} e_{1}\right)(x)=v_{n, q}(x), \quad\left(S_{n, q}^{*} e_{2}\right)(x)=x^{2}, \\
\left(S_{n, q}^{*} \psi_{x}^{2}\right)(x)=2 x\left(x-v_{n, q}(x)\right),
\end{gathered}
$$

where $\psi_{x}(t)=t-x, t \geq 0$.

Since the identities are easily obtained by direct computation, we omit the proof.

Examining relations (2.4), (2.6) and based on Bohman-Korovkin theorem, it is clear that $\left(S_{n, q}^{*}\right)_{n \geq 1}$ does not form an approximation process. The next step is to 
transform it for enjoying of this property. For each $n \in \mathbb{N}$, the constant $q$ will be replaced by a number $q_{n} \in(0,1)$ such that $\lim _{n} q_{n}=1$. At this stage we also need a connection between the involved sequences $\left(b_{n}\right)_{n \geq 1},\left(q_{n}\right)_{n \geq 1}$.

Theorem 2.2. Let $\left(q_{n}\right)_{n \geq 1}, 0<q_{n}<1$, be a sequence and let the operators $S_{n, q_{n}}^{*}, n \in \mathbb{N}$, be defined as in (2.5). If

$$
\lim _{n} q_{n}=1, \quad \lim _{n} \frac{b_{n}}{1-q_{n}^{n}}=\infty \quad \text { and } \quad \lim _{n} \frac{b_{n}}{[n]_{q_{n}}}=0
$$

then for any compact $K \subset \mathbb{R}_{+}$and for each $f \in C\left(\mathbb{R}_{+}\right)$one has

$$
\lim _{n}\left(S_{n, q_{n}}^{*} f\right)(x)=f(x), \text { uniformly in } x \in K .
$$

Proof. The second limit in (2.8) guarantees that $\bigcup_{n=1}^{\infty} J_{n}\left(q_{n}\right)=\mathbb{R}_{+}$. Consequently, the sequence of operators is proper defined, this meaning that it is suitable to approximate functions defined on $\mathbb{R}_{+}$. The third limit in (2.8) implies $\lim _{n} v_{n, q_{n}}(x)=x^{2}$ uniformly in $x \in K$. The result follows from Bohman-Korovkin $n$
criterion via Lemma 2.1 .

Remark 2.3. For removing any doubt, we indicate pairs of sequences $\left(q_{n}\right)_{n \geq 1}$, $\left(b_{n}\right)_{n \geq 1}$ which verify the plurality of requirements imposed in Theorem 2.2.

$$
\begin{aligned}
& 1^{\circ} q_{1}=\frac{1}{\sqrt{2}} \text { and } q_{n}=\frac{1}{\sqrt[n]{n}}(n \geq 2) ; b_{n}=n^{\lambda} \text { for any fixed } \lambda \in\left(0, \frac{1}{2}\right) . \\
& 2^{\circ} q_{1}=\frac{1}{2} \text { and } q_{n}=1-\frac{1}{n}(n \geq 2) ; b_{n}=\left([n]_{q_{n}}\right)^{\lambda} \text { for any fixed } \lambda \in(0,1) .
\end{aligned}
$$

\section{Weighted Statistical Approximation Property}

A real valued function $\rho$ defined on $\mathbb{R}$ is usually called a weight function if it is continuous on the domain satisfying the conditions $\rho \geq e_{0}$ and $\lim _{|x| \rightarrow \infty} \rho(x)=\infty$. For example, the mapping $x \mapsto 1+x^{2+\lambda}, \lambda$ a non-negative parameter, is often used as weight function. Let consider the spaces

$$
\begin{gathered}
B_{\rho}(\mathbb{R})=\left\{f: \mathbb{R} \rightarrow \mathbb{R} \mid \text { a constant } M_{f} \text { depending on } f\right. \text { exists } \\
\left.\quad \text { such that }|f| \leq M_{f} \rho\right\}, \\
C_{\rho}(\mathbb{R})=\left\{f \in B_{\rho}(\mathbb{R}) \mid f \text { continuous on } \mathbb{R}\right\},
\end{gathered}
$$


endowed with the usual norm $\|\cdot\|_{\rho}$, this meaning

$$
\|f\|_{\rho}=\sup _{x \in \mathbb{R}} \frac{|f(x)|}{\rho(x)} .
$$

O. Duman and C. Orhan proved [9, Theorem 3] the following weighted Korovkin type theorem via A-statistical convergence.

Theorem A. Let $A=\left(a_{n, k}\right)$ be a non-negative regular summability matrix and let $\bar{\rho}_{1}, \bar{\rho}_{2}$ weight functions such that

$$
\lim _{|x| \rightarrow \infty} \frac{\bar{\rho}_{1}(x)}{\bar{\rho}_{2}(x)}=0 .
$$

Assume that $\left(T_{n}\right)_{n \geq 1}$ is a sequence of positive linear operators from $C_{\bar{\rho}_{1}}(\mathbb{R})$ into $B_{\bar{\rho}_{2}}(\mathbb{R})$. One has

$$
s t_{A}-\lim _{n}\left\|T_{n} f-f\right\|_{\bar{\rho}_{2}}=0, \quad f \in C_{\bar{\rho}_{1}}(\mathbb{R}),
$$

if and only if

$$
s t_{A}-\lim _{n}\left\|T_{n} F_{k}-F_{k}\right\|_{\bar{\rho}_{1}}=0, \quad k=0,1,2,
$$

where $F_{k}(x)=x^{k} \bar{\rho}_{1}(x) /\left(1+x^{2}\right)$.

Clearly, all the above notations and results are still valid if we replace the domain $\mathbb{R}$ by $\mathbb{R}_{+}$. The main result of this section is based on Theorem $A$. We choose the pair of weight functions $\left(\rho_{0}, \rho_{\lambda}\right)$, where

$$
\rho_{0}(x)=1+x^{2}, \quad \rho_{\lambda}(x)=1+x^{2+\lambda}, \quad x \in \mathbb{R}_{+},
$$

$\lambda>0$ being a fixed parameter. Relation (3.1) is fulfilled and $B_{\rho_{0}}\left(\mathbb{R}_{+}\right) \subset B_{\rho_{\lambda}}\left(\mathbb{R}_{+}\right)$. Moreover, taking $A$ the Cesaro matrix of first order, Theorem A implies

Corollary 3.1. For any sequence $\left(T_{n}\right)_{n \geq 1}$ of linear positive operators acting from $C_{\rho_{0}}\left(\mathbb{R}_{+}\right)$into $C_{\rho_{\lambda}}\left(\mathbb{R}_{+}\right), \lambda>0$, one has

$$
s t-\lim _{n}\left\|T_{n} f-f\right\|_{\rho_{\lambda}}=0, \quad f \in C_{\rho_{0}}\left(\mathbb{R}_{+}\right),
$$

if and only if

$$
s t-\lim _{n}\left\|T_{n} e_{k}-e_{k}\right\|_{\rho_{0}}=0, \quad k=0,1,2 .
$$

Next, we collect some elementary properties of the functions defined by (2.4). 
Lemma 3.2. Let $v_{n, q}, n \in \mathbb{N}$, be defined by (2.4), where $q \in(0,1)$ and $b_{n}>0$, $n \in \mathbb{N}$. The following statements are true.

$$
\begin{gathered}
v_{n, q}(0)=0, \quad v_{n, q}\left(\frac{b_{n}}{1-q^{n}}\right)=\frac{b_{n}}{1-q^{n}} ; \\
0 \leq v_{n, q}(x) \leq x, \quad x \in\left[0, \frac{b_{n}}{1-q^{n}}\right] ; \\
x-v_{n, q}(x) \leq x_{0}-v_{n, q}\left(x_{0}\right) \leq \frac{b_{n}}{2 q[n]_{q}}, \quad x \geq 0,
\end{gathered}
$$

where $x_{0}=\frac{b_{n}}{2[n]_{q} \sqrt{1-q}}$.

Proof. Both (i) and (ii) are obtained by a straightforward calculation. Because $S_{n, q}^{*}$ is a positive operators, actually, the inequality $v_{n, q}(x) \leq x$ springs from (2.7).

For proving (iii) we can consider the function $h:[0, \infty) \rightarrow \mathbb{R}, h(x)=x-$ $v_{n, q}(x)$. The unique solution of the equation $\frac{d}{d x} h(x)=0$ being $x_{0}$, the monotonicity of $h$ implies $h(x) \leq h\left(x_{0}\right)=\frac{b_{n}(1-\sqrt{1-q})}{2 q[n]_{q}}$ and (3.6) follows.

The main result of this section will be read as follows.

Theorem 3.3. Let the sequence $\left(q_{n}\right)_{n \geq 1}, 0<q_{n}<1$, be given such that st $-\lim _{n} q_{n}=1$. Let the operators $S_{n, q_{n}}^{*}, n \in \mathbb{N}$, be defined as in (2.5). If

$$
s t-\lim _{n} \frac{b_{n}}{[n]_{q_{n}}}=0,
$$

then, for each function $f \in C_{\rho_{0}}\left(\mathbb{R}_{+}\right)$, one has

$$
s t-\lim _{n}\left\|S_{n, q_{n}}^{*} f-f\right\|_{\rho_{\lambda}}=0,
$$

where $\lambda>0$.

Proof. Each function $S_{n, q_{n}}^{*} f, f \in C_{\rho_{0}}\left(\mathbb{R}_{+}\right)$, is defined on $J_{n}\left(q_{n}\right)$ (see (2.5)). We extend it on $\mathbb{R}_{+}$in the classical manner. Let $\widetilde{S}_{n, q_{n}}^{*}$ be defined as follows

$$
\left(\widetilde{S}_{n, q_{n}}^{*} f\right)(x)=\left\{\begin{array}{cl}
\left(S_{n, q_{n}}^{*} f\right)(x), & x \in J_{n}\left(q_{n}\right), \\
f(x), & x \geq \frac{b_{n}}{1-q_{n}^{n}} .
\end{array}\right.
$$


For each $n \in \mathbb{N}$, the norm $\left\|\widetilde{S}_{n, q_{n}}^{*} f-f\right\|_{\rho_{\lambda}}$ coincides with the norm of the element $\left(S_{n, q_{n}}^{*} f-f\right)$ in the space $B_{\rho_{\lambda}}\left(J_{n}\left(q_{n}\right)\right)$, for any $\lambda \geq 0$. Applying Corollary 3.1 to the operators $T_{n} \equiv \widetilde{S}_{n, q_{n}}^{*}$, the proof of Theorem 3.3 will be finished. In this respect, it is sufficient to prove that, under our hypothesis, the operators verify the conditions given at (3.4).

By the first and the third identity of relation (2.6) it is clear that

$$
s t-\lim _{n}\left\|\widetilde{S}_{n, q_{n}}^{*} e_{k}-e_{k}\right\|_{\rho_{0}}=0
$$

for $k=0$ and $k=2$. The second identity of (2.6) and Lemma 3.2 allow us to write

$$
\begin{gathered}
\sup _{x \in J_{n}\left(q_{n}\right)} \frac{1}{\rho_{0}(x)}\left|\left(S_{n, q_{n}}^{*} e_{1}\right)(x)-x\right|=\sup _{x \in J_{n}\left(q_{n}\right)} \frac{x-v_{n, q_{n}}(x)}{1+x^{2}} \\
\leq \sup _{x \geq 0} \frac{x-v_{n, q_{n}}(x)}{1+x^{2}} \leq \frac{b_{n}}{2 q_{n}[n]_{q_{n}}} .
\end{gathered}
$$

Since $s t-\lim _{n} q_{n}=1$, based on (3.7) we get $s t-\lim _{n}\left\|\widetilde{S}_{n, q_{n}}^{*} e_{1}-e_{1}\right\|_{\rho_{0}}=0$ and the proof is completed.

\section{Rate of Weighted Approximation}

The $q$-Stirling numbers of the second kind denoted by $\mathcal{S}_{q}(m, k)\left(m, k \in \mathbb{N}_{0}\right.$, $m \geq k)$ are described by the recurrence formula

$$
\mathcal{S}_{q}(m, k)=\mathcal{S}_{q}(m-1, k-1)+[k]_{q} \mathcal{S}_{q}(m-1, k), \quad m \geq k \geq 1,
$$

with $\mathcal{S}_{q}(0,0)=1$ and $\mathcal{S}_{q}(m, 0)=0$ for $m \in \mathbb{N}$. We agree $\mathcal{S}_{q}(m, k)=0$ for $k>m$. The closed form is the following

$$
\mathcal{S}_{q}(m, k)=\frac{1}{[k]_{q} ! q^{\frac{k(k-1)}{2}}} \sum_{j=0}^{k}(-1)^{j} q^{\frac{j(j-1)}{2}}\left[\begin{array}{l}
k \\
j
\end{array}\right]_{q}^{m}[k-j]_{q}^{m}, \quad 1 \leq k \leq m .
$$

For $q \rightarrow 1^{-}, \mathcal{S}_{1}(m, k)$ represents the number of ways of partitioning a set of $m$ elements into $k$ non-empty subsets [1, p. 824].

Lemma 4.1. Let the sequence $\left(q_{n}\right)_{n \geq 1}, 0<q_{n}<1$, be given and let the operators $S_{n, q_{n}}^{*}, n \in \mathbb{N}$, be defined as in (2.5). One has

$$
\left(S_{n, q_{n}}^{*} e_{m}\right)(x)=q_{n}^{\frac{m(m-1)}{2}} v_{n, q_{n}}^{m}(x)+\sum_{k=1}^{m-1}\left(\frac{b_{n}}{[n]_{q_{n}}}\right)^{m-k} \mathcal{S}_{q_{n}}(m, k) q_{n}^{\frac{k(k-1)}{2}} v_{n, q_{n}}^{k}(x),
$$


$x \in J_{n}\left(q_{n}\right)$, where $\mathcal{S}_{q_{n}}(m, k)$ are $q_{n}$-Stirling numbers given by (4.1). Here $e_{m}$ stands for the monomial of $m$ degree.

Proof. Taking in view both (2.5) and (2.1) and using the Cauchy rule (or Mertens formula) for multiplication of two series, we can write

$$
\begin{aligned}
\left(S_{n, q_{n}}^{*} e_{m}\right)(x) & =\sum_{k=0}^{\infty} \sum_{i=0}^{k}(-1)^{i} q_{n}^{\frac{i(i-1)}{2}} e_{m}\left(\frac{[k-i]_{q_{n}} b_{n}}{[n]_{q_{n}}}\right) \frac{\left([n]_{q_{n}} v_{n, q_{n}}(x)\right)^{k}}{[i]_{q_{n}} ![k-i]_{q_{n}} ! b_{n}^{k}} \\
& =\sum_{k=0}^{\infty} \mathcal{S}_{q_{n}}(m, k)[k]_{q_{n}} ! q_{n}^{\frac{k(k-1)}{2}}\left(\frac{b_{n}}{[n]_{q_{n}}}\right)^{m-k} \cdot \frac{1}{[k]_{q_{n}} !} v_{n, q_{n}}^{k}(x) \\
& =\sum_{k=0}^{m}\left(\frac{b_{n}}{[n]_{q_{n}}}\right)^{m-k} \mathcal{S}_{q_{n}}(m, k) q_{q^{\frac{k(k-1)}{2}}} v_{n, q_{n}}^{k}(x) \\
& =\mathcal{S}_{q_{n}}(m, m) q_{n}^{\frac{m(m-1)}{2}} v_{n, q_{n}}^{m}(x) \\
& +\sum_{k=0}^{m-1}\left(\frac{b_{n}}{[n]_{q_{n}}}\right)^{m-k} \mathcal{S}_{q_{n}}(m, k) q_{n}^{\frac{k(k-1)}{2}} v_{n, q_{n}}^{k}(x) .
\end{aligned}
$$

Knowing that $\mathcal{S}_{q_{n}}(m, m)=1$ and $\mathcal{S}_{q_{n}}(m, 0)=0(m \geq 1)$, one obtains (4.1).

We mention that A. Aral proved a similar result [3, Lemma 1] for the operators given at (2.3). His proof is based on the forward $q$-differences up to order $m$.

Set $A_{m}\left(n ; q_{n}, b_{n}\right):=\sum_{k=1}^{m-1} \mathcal{S}_{q_{n}}(m, k)\left(\frac{b_{n}}{[n]_{q_{n}}}\right)^{m-k}$.

Under the hypothesis $\lim _{n} q_{n}=1$ and $\lim _{n} \frac{b_{n}}{[n]_{q_{n}}}=0$ we get $\lim _{n} \mathcal{S}_{q_{n}}(m, k)=$ $\mathcal{S}_{1}(m, k), 1 \leq k \leq m-1$, and a real constant $B_{m}$ depending only on $m$ exists such that

$$
\sup _{n \in \mathbb{N}} A_{m}\left(n ; q_{n}, b_{n}\right)=B_{m}
$$

Lemma 4.2. Let the sequence $\left(q_{n}\right)_{n \geq 1}, 0<q_{n}<1$, be given and let $S_{n, q_{n}}^{*}$, $n \in \mathbb{N}$, be operators defined as in (2.5).

If the conditions (2.8) are fulfilled, then one has

$$
\left(S_{n, q_{n}}^{*} e_{m}\right)(x) \leq\left(1+B_{m}\right)\left(1+x^{m}\right), \quad x \in J_{n}\left(q_{n}\right),
$$

where $B_{m}$ is given at (4.2). 
(ii) For each $n \in \mathbb{N}$, the operator $S_{n, q_{n}}^{*}$ maps the space $B_{\rho_{\lceil\lambda]}}$ into $B_{\rho_{\lceil\lambda\rceil}}, \lambda>0$. Here $\lceil\lambda\rceil$ represents the ceiling of number $\lambda$.

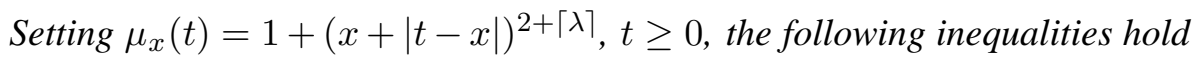

$$
S_{n, q_{n}}^{*} \mu_{x} \leq c_{\lambda}\left(1+e_{2+\lceil\lambda\rceil}\right)
$$

$$
\sqrt{S_{n, q_{n}}^{*} \mu_{x}^{2}} \leq \widetilde{c}_{\lambda}\left(1+e_{2+\lceil\lambda\rceil}\right)
$$

where $c_{\lambda}, \widetilde{c}_{\lambda}$ are constants independent on $x$ and $n$.

\section{Proof.}

(i) Based on Lemma 4.1, relation (3.5) and knowing that $q_{n} \in(0,1)$, for each $x \in J_{n}\left(q_{n}\right)$ we can write

$$
\begin{aligned}
\left(S_{n, q_{n}}^{*} e_{m}\right)(x) & \leq x^{m}+\sum_{k=1}^{m-1} \mathcal{S}_{q_{n}}(m, k)\left(\frac{b_{n}}{[n]_{q_{n}}}\right)^{m-k} x^{k} \\
& \leq x^{m}+m A_{m}\left(n ; q_{n}, b_{n}\right)\left(1+x^{m}\right) \\
& \leq x^{m}+m B_{m}\left(1+x^{m}\right),
\end{aligned}
$$

and (4.3) follows.

(ii) If $f \in B_{\rho_{\lceil\lambda\rceil}}$, then $|f| \leq M_{f}\left(1+x^{2+\lceil\lambda\rceil}\right)$. $S_{n, q_{n}}^{*}$ being linear and positive is monotone. Relation (4.3) implies our statement.

(iii) For each $t \geq 0$ and $x \in J_{n}\left(q_{n}\right)$ we get

$$
\mu_{x}(t) \leq 1+(2 x+t)^{2+\lceil\lambda\rceil} \leq 1+2^{1+\lceil\lambda\rceil}\left((2 x)^{2+\lceil\lambda\rceil}+t^{2+\lceil\lambda\rceil}\right) .
$$

By using (4.3) and (2.6) we obtain (4.4).

(iv) Since $\mu_{x}^{2}(t) \leq 2\left(1+(2 x+t)^{4+2\lceil\lambda\rceil}\right)$, the same relations (4.3) and (2.6) imply (4.5).

We proceed with estimated of the errors $\left|S_{n, q_{n}}^{*} f-f\right|, n \in \mathbb{N}$, involving unbounded functions by using a weighted modulus of smoothness associated to the space $B_{\rho_{\lambda}}$. In this respect we use

$$
\Omega_{\rho_{\lambda}}(f ; \delta)=\sup _{\substack{x \geq 0 \\ 0<h \leq \delta}} \frac{|f(x+h)-f(x)|}{1+(x+h)^{2+\lambda}}, \quad \delta>0 .
$$


This modulus was recently considered by Lopez-Moreno [17].

Clearly, $\Omega_{\rho_{\lambda}}(f ; \cdot) \leq 2\|f\|_{\rho_{\lambda}}$ for each $f \in B_{\rho_{\lambda}}$. Among some basic properties of this modulus, in [17] the following is mentioned: $\Omega_{\rho_{\lambda}}(f ; m \delta) \leq m \Omega_{\rho_{\lambda}}(f ; \delta)$ for any positive integer $m$.

Since $\Omega_{\rho_{\lambda}}(f ; \delta)$ is monotonically increasing with respect to $\delta(\delta>0)$ and $\alpha<[\alpha]+1 \leq \alpha+1$ holds ( $[\alpha]$ gives the integer part of $\alpha$ ), the following inequality

$$
\Omega_{\rho_{\lambda}}(f ; \alpha \delta) \leq(\alpha+1) \Omega_{\rho_{\lambda}}(f ; \delta), \quad \delta>0,
$$

is valid for any $\alpha>0$.

Theorem 4.3. Let the sequence $\left(q_{n}\right)_{n \geq 1}, 0<q_{n}<1$, be given and let $S_{n, q_{n}}^{*}$, $n \in \mathbb{N}$, be operators defined as in (2.5) such that the conditions (2.8) are fulfilled. For each $f \in B_{\rho_{[\lambda]}}$ the following inequality

$$
\left|\left(S_{n, q_{n}}^{*} f\right)(x)-f(x)\right| \leq k_{\lambda}\left(1+x^{3+\lceil\lambda\rceil}\right) \Omega_{\rho_{\lceil\lambda\rceil}}\left(f ; \sqrt{\frac{b_{n}}{[n]_{q_{n}}}}\right), \quad x \in J_{n}\left(q_{n}\right),
$$

holds, where $k_{\lambda}$ is a constant independent of $f$ and $n$.

Proof. Let $n \in \mathbb{N}$ and $f \in B_{\rho_{\lceil\lambda]}}$ be fixed. For each $t \geq 0$ and $\delta>0$, based both on definition (4.6) and on property (4.7) with $\alpha:=|t-x| \delta^{-1}$, we get

$$
\begin{aligned}
|f(t)-f(x)| & \leq\left(1+(x+|t-x|)^{2+\lceil\lambda\rceil}\right)\left(\frac{|t-x|}{\delta}+1\right) \Omega_{\rho_{\lceil\lambda\rceil}}(f ; \delta) \\
& =\left(\mu_{x}(t)+\frac{1}{\delta} \mu_{x}(t)|t-x|\right) \Omega_{\rho_{\lceil\lambda]}(f ; \delta),}
\end{aligned}
$$

where $\mu_{x}$ was introduced at Lemma 4.2.

Taking into account that $S_{n, q_{n}}^{*}$ is linear positive operator preserving the constants, we can write

$$
\begin{aligned}
& \left|\left(S_{n, q_{n}}^{*} f\right)(x)-f(x)\right|=\left|S_{n, q_{n}}^{*}(f-f(x) ; x)\right| \leq S_{n, q_{n}}^{*}(|f-f(x)| ; x) \\
\leq & S_{n, q_{n}}^{*}\left(\mu_{x}+\delta^{-1} \mu_{x}\left|\psi_{x}\right| ; x\right) \Omega_{\rho_{\lceil\lambda\rceil}}(f ; \delta) \\
= & \left\{\left(S_{n, q_{n}}^{*} \mu_{x}\right)(x)+\frac{1}{\delta}\left(S_{n, q_{n}}^{*} \mu_{x}\left|\psi_{x}\right|\right)(x)\right\} \Omega_{\rho_{\lceil\lambda\rceil}}(f ; \delta) \\
\leq & \left\{\left(S_{n, q_{n}}^{*} \mu_{x}\right)(x)+\frac{1}{\delta} \sqrt{\left(S_{n, q_{n}}^{*} \mu_{x}^{2}\right)(x)} \sqrt{\left(S_{n, q_{n}}^{*} \psi_{x}^{2}\right)(x)}\right\} \Omega_{\rho_{\lceil\lambda\rceil}}(f ; \delta),
\end{aligned}
$$

where $\psi_{x}$ was introduced at Lemma 2.1. 
The last increase is based on Cauchy-Schwarz inequality frequent used for positive operators of discrete type. It was proved by Yuan-Chuan Li and Sen-Yen Shaw [6] that this classical inequality has great and unexpected force.

Relations (2.7) and (3.6) allow us to write $\left(S_{n, q_{n}}^{*} \psi_{x}^{2}\right)(x) \leq \frac{b_{n} x}{[n]_{q_{n}}}$. Further on, by using inequalities (4.4), (4.5) we get

$$
\begin{aligned}
& \left|\left(S_{n, q_{n}}^{*} f\right)(x)-f(x)\right| \\
\leq & \left\{c_{\lambda}\left(1+x^{2+\lceil\lambda\rceil}\right)+\frac{\widetilde{c}_{\lambda}}{\delta}\left(1+x^{2+\lceil\lambda\rceil}\right) \sqrt{x} \sqrt{\frac{b_{n}}{[n]_{q_{n}}}}\right\} \Omega_{\rho_{\lceil\lambda\rceil}}(f ; \delta) \\
\leq & 2\left(c_{\lambda}+\frac{\widetilde{c}_{\lambda}}{\delta} \sqrt{\frac{b_{n}}{[n]_{q_{n}}}}\right)\left(1+x^{3+\lceil\lambda\rceil}\right) \Omega_{\rho_{\lceil\lambda\rceil}}(f ; \delta) .
\end{aligned}
$$

Choosing $\delta=\sqrt{\frac{b_{n}}{[n]_{q_{n}}}}$ and setting $k_{\lambda}:=2\left(c_{\lambda}+\widetilde{c}_{\lambda}\right)$, the conclusion follows.

Corollary 4.4. Under the assumptions of Theorem 4.3 the following global estimate takes place

$$
\left\|S_{n, q_{n}}^{*} f-f\right\|_{\rho_{\lceil\lambda+1]}} \leq k_{\lambda} \Omega_{\rho_{\lceil\lambda]}}\left(f ; \sqrt{\frac{b_{n}}{[n]_{q_{n}}}}\right), \quad f \in B_{\rho_{\lceil\lambda\rceil}} .
$$

\section{REFERENCES}

1. M. Abramowitz and I. A. Stegun (eds.), Handbook of Mathematical Functions with Formulas, Graphs and Mathematical Tables, National Bureau of Standards Appplied Mathematics, Series 55, Issued June, 1964.

2. O. Agratini, Linear operators that preserve some test functions, Int. J. of Math. and Math. Sci., Art. ID 94136, (2006), 1-11.

3. A. Aral, A generalization of Szász-Mirakyan operators based on $q$-integers, Mathematical and Computer Modelling, 47 (2008), 1052-1062.

4. A. Aral and O. Doğru, Bleimann, Butzer and Hahn operators based on the $q$-integers, J. Ineq. and Appl., Art. ID 79410, (2007), 1-12.

5. A. Aral and V. Gupta, The $q$-derivative and applications to $q$-Szász Mirakyan operators, Calcolo, 43 (2006), 151-170.

6. Yuan-Chuan Li and Sen-Yen Shaw, A proof of Hölder's inequality using the CauchySchwarz inequality, Journal of Inequalities in Pure and Applied Mathematics, 7 (2006), Issue 2, Article 62, 1-3. 
7. M.-M. Derriennic, Modified Bernstein polynomials and Jacobi polynomials in $q$ calculus, Rendiconti del Circolo Matematico di Palermo, Serie II, Suppl., 76 (2005), 269-290.

8. O. Doğru and O. Duman, Statistical approximation of Meyer-König and Zeller operators based on the $q$-integers, Publ. Math. Debrecen, 68 (2006), 199-214.

9. O. Duman and C. Orhan, Statistical approximation by positive linear operators, Studia Math., 161(2) (2004), 187-197.

10. H. Fast, Sur la convergence statistique, Colloq. Math., 2 (1951), 241-244.

11. J. A. Fridy and H. I. Miller, A matrix characterization of statistical convergence, Analysis, 11 (1991), 59-66.

12. A. D. Gadjiev and C. Orhan, Some approximation theorems via statistical convergence, Rocky Mountain J. Math., 32(1) (2002), 129-138.

13. G. Gasper and M. Rahman, Basic hypergeometric series, Cambridge Univ. Press, 1990.

14. V. Kac and P. Cheung, Quantum Calculus, Universitext, Springer, 2002.

15. J. P. King, Positive linear operators which preserve $x^{2}$, Acta Math. Hungar., 99(3) (2003), 203-208.

16. E. Kolk, Matrix summability of statistically convergent sequences, Analysis, (1993), 77-83.

17. A.-J. López-Moreno, Weighted simultaneous approximation with Baskakov type operators, Acta Mathematica Hungarica, 104(1-2) (2004), 143-151.

18. A. Lupaş, A $q$-analogue of the Bernstein operator, University of Cluj-Napoca, Seminar on Numerical and Statistical Calculus, 9 (1987), 85-92.

19. S. Ostrovska, $q$-Bernstein polynomials and their iterates, J. Approx. Th., 123 (2003), 232-255.

20. S. Ostrovska, On the Lupaş $q$-analogue of the Bernstein operator, Rocky Mountain Journal of Mathematics, 36(5) (2006), 1615-1629.

21. G. M. Phillips, Bernstein polynomials based on the $q$-integers, Ann. Numer. Math., 4 (1997), 511-518.

22. T. Trif, Meyer-Konig and Zeller operators based on the $q$-integers, Rev. Anal. Numer. Théor. Approx., 29 (2000), 221-229.

23. V. S. Videnskii, On some classes of $q$-parametric positive operators, Operator Theory Adv. Appl., 158 (2005), 213-222.

24. H. Wang, Korovkin-type theorem and application, J. Approx. Th., 132(2) (2005), 258-264. 
Octavian Agratini

Faculty of Mathematics and Computer Science,

Babeş-Bolyai University,

400084 Cluj-Napoca,

Romania

E-mail: agratini@math.ubbcluj.ro

Ogün Doğru

Department of Mathematics,

Faculty of Sciences and Arts,

Gazi University,

Teknikokullar 06500,

Ankara,

Turkey

E-mail: ogun.dogru@gazi.edu.tr 